\title{
CASTEL DE CÀLLER NEL MEDITERRANEO DEL SECOLO XIV: COMMERCIO E SOCIETÀ URBANA NELLA CORONA D'ARAGONA
}

\author{
CASTEL DE CÀLLER IN THE MEDITERRANEAN SEA OF 14TH CENTURY: \\ TRADE AND URBAN SOCIETY OF THE CROWN OF ARAGON
}

Simonetta Figus

Universidad de Zaragoza

\begin{abstract}
Resumen: El objetivo de este trabajo pretende reflexionar sobre los libros de cuentas de una familia de hombres de negocios de Barcelona en la primera mitad del 1300 y sus relaciones con la ciudad de Cagliari (llamada en las fuentes Castel de Càller) vista como uno de los principales centros de actividades comercial del Mediterráneo Occidental
\end{abstract}

Palabras clave: Castel de Càller, Mediterráneo, libros de cuentas, mercaderes.

\begin{abstract}
This text aims to reflect on the books of account of a traders family from Barcelona in the first half of 14th century and its relationships with the city of Cagliari, as one of the main commercial activities centers of the western Mediterranean.
\end{abstract}

Key words: city, Mediterranean sea, account books, traders. 


\section{Introduzione}

Negli ultimi anni sono notevolmente aumentate le conoscenze sui rapporti commerciali ed economici tra la Sardegna e il mondo iberico nel XIV secolo. Si tratta di un periodo cruciale per la storia sarda, profondamente segnata dalla conquista aragonese, i cui sovrani, ottenuto nel 1297, da papa Bonifacio VIII, il Regnum Sardiniae et Corsicae, incorporarono la Sardegna all'interno della Corona d'Aragona, la confederazione di regni e signorie che formava il loro vasto dominio mediterraneo (Salavert y Roca, 1956: 114-125; Arribas Palau, 1952: 55; Casula, 1990: 61-70; Cadeddu, 2007:149-156; Sanna, 2008: 737-752).

La Sardegna da iniziale rotta d'interesse commerciale divenne, dopo il 1297, una conquista politica sempre più necessaria per i re d'Aragona; infatti, oltre a uno scalo marittimo e commerciale importante, l'isola era anche una base necessaria per i suoi traffici nel bacino del Mediterraneo; questi ultimi riguardavano un'ampia porzione di territorio compreso fra le coste nord africane e quelle tirreniche (Tangheroni, 1989: 50-65; 1981; 1983: 43-54; Manca, 1969; 1966; 1967)

La conquista ebbe inizio nel 1323 dopo un lungo periodo di trattative con Pisa che controllava gran parte della Sardegna e in particolare il centro più importante Castel di Castro, nome pisano della città di Cagliari, che fu definitivamente strappata ai pisani nel $1326 .{ }^{1}$ Dopo tale data, i catalanoaragonesi si insediarono stabilmente nella città, divenuta, con i nuovi dominatori, Castel de Càller, trasferendosi dalla vicina cittadella fortificata di Bon Ayre. ${ }^{2}$ Nulla fu sostanzialmente modificato nell'assetto cittadino tranne il quartiere del porto che fu reimpiantato urbanisticamente secondo canoni tipicamente catalani (Cadinu, 2009: 81-101).

1 Tra la fine del Duecento e l'inizio del Trecento il comune di Pisa aveva il controllo diretto di quello che fu il giudicato di Cagliari (esso ebbe fine con la distruzione del suo centro più importante, Santa Igia, nel 1257 ad opera dei pisani), del giudicato di Gallura sottratto ai Visconti e in seguito della città di Iglesias già appartenente ai conti di Donoratico, come la sesta parte del cagliaritano. La venuta dei pisani in Sardegna, unitamente ai genovesi, aveva comportato un notevole mutamento nelle condizioni politiche ed economiche dell'isola. I mercanti provenienti dalla madrepatria si stabilirono nell'isola e diedero luogo a comunità organizzate ed efficienti, isolate dalla popolazione locale. Così sorsero o si trasformarono le città nell'isola favorite dal comune d'origine, con propri ordinamenti e statuti mercantili.

2 Si tratta del grande agglomerato-città, fondato dagli aragonesi dell'infante Alfonso, sorto sul colle di Bonaria e funzionale all'assedio di Castel di Castro. Fu popolato con almeno 5000 individui e le sue mura distavano meno di 450 metri dalle mura delle appendici del Castello. 


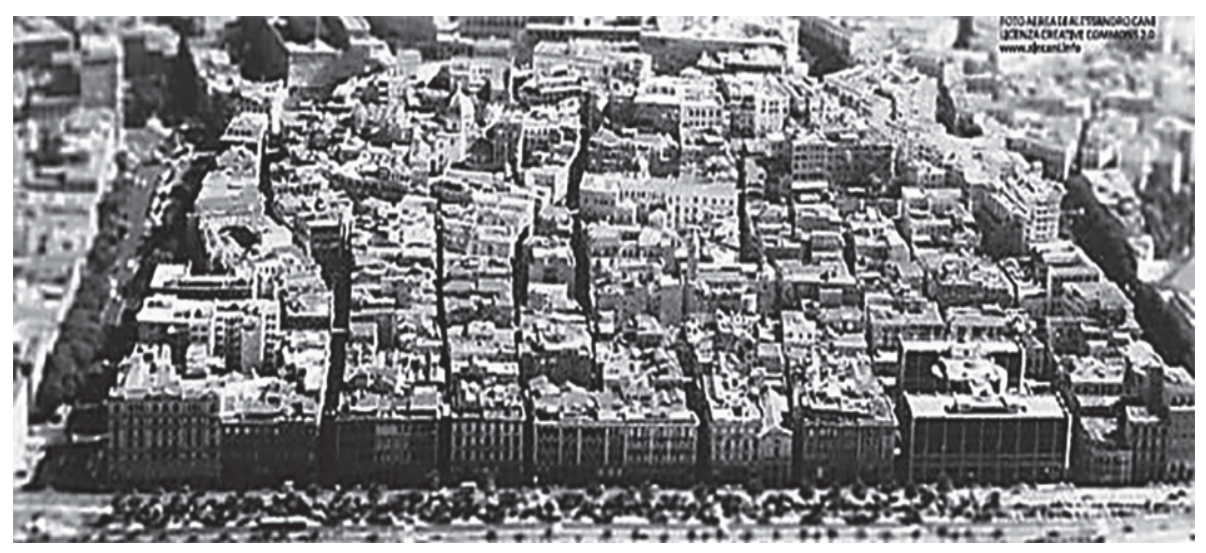

Figura 1 1-Veduta d'insieme dell'appendice della Lapola.

È evidente il tracciato di impianto aragonese. (Fonte: Google Earth).

Era questo un punto nevralgico di grande importanza per i traffici commerciali della città, funzionale al ruolo che la Corona intendeva imprimere all'isola. ${ }^{3}$

\section{I libri di conto della famiglia Benet ${ }^{4}$}

Per affrontare lo studio della realtà socioeconomica della città e dei suoi legami con la Corona ci siamo serviti di una serie di libri di conto di una famiglia della media borghesia catalana. ${ }^{5}$ I Benet di Barcellona costituirono una

3 Nel Breve Portus Kallaretani, regolamento del porto della città scritto dai pisani tra il 1317-18, è attestata la fervente attività del porto di Bagnaria di Castel di Castro. Nei documenti della medesima epoca compare però anche il termine Lapola, destinato a diventare il nome definitivo del borgo marinaro, finché in epoca moderna si affermò quello di Marina. Sull'origine del toponimo Bagnaria, sono determinanti le scoperte archeologiche avvenute in situ, probabilmente il nome è legato ai resti di imponenti infrastrutture idriche d'epoca classica.

4 Il presente lavoro prende spunto dalla tesi di laurea magistrale realizzata nell'anno accademico 2012 2013, I commerci tra Barcellona e Cagliari, tesi di laurea magistrale di Simonetta Figus, relatore prof. Sergio Tognetti, Università degli Studi di Cagliari. Il lavoro sui rimanenti libri di conto della famiglia Benet proseguirà nella ricerca in corso per il Dottorato in Historia, Sociedad y Cultura: Épocas Medieval y Moderna presso 1'Università di Saragozza, sotto la direzione di Prof. German Navarro Espinach e dott. Luciano Gallinari.

5 Archivo Capitular de Barcelona (da ora ACB), Llibres extravagants, Comptes de particulars, Llibre de deu y deig di Joan Benet 1334-1338. ACB, Llibres extravagants, Comptes de particulars, Manual de comptes di Johan Benet, 1338-1344. ACB, Llibres extravagants, Comptes de particulars, Llibre de comptes di Joan Bonet, 1332-1356. 
compagnia mercantile che possedeva filiali in tutto il bacino del Mediterraneo e che operò attivamente per tutta la prima metà del xIV secolo attraverso figli, generi e nipoti. La loro attività fu bruscamente interrotta dall'epidemia di peste del 1348 che vide la morte di quasi tutti i suoi membri più influenti.

I libri di conto, in fase di studio, costituiscono la base di partenza su cui costruire uno studio tra la città di Castel de Càller e le altre realtà della Corona con cui entrò in contatto per motivi economici e commerciali.

I libri conservati nell'archivio capitolare di Barcellona e aventi relazione con la città sarda sono tre:

Il llibre de deu i deg di Johan Benet 1334-1338, il Manual de comptes di Johan Benet, 1338-1344 e il Llibre de comptes di Joan Bonet, 1332-1356. Ho avuto occasione di studiare il primo di questi libri (llibre de deu i deg di Johan Benet) durante la mia tesi di laurea discussa nel 2013.

Esso documenta la permanenza di Johan Benet a Castel de Càller negli anni dal 1334 al 1338. Da tale fonte si sono potute trarre informazioni importanti sulla città e sulle sue relazioni commerciali con il bacino del Mediterraneo durante i primi anni della dominazione aragonese in Sardegna.

Un altro registro scritto da Johan Benet era già stato studiato in precedenza da Maria Marsá confermando l'importanza della famiglia nei rapporti commerciali tra l'Aragona e la Sardegna ${ }^{6}$ (Marsá, 1977). Da tale studio abbiamo appreso che Johan risiedette a Castel de Càller fin dal 1332, cioè dopo appena sei anni dalla conquista dell'isola.

Lo studio della famiglia Benet, nel momento della sua massima espansione di interessi in tutto il bacino del Mediterraneo, si è rivelato importante per approfondire i particolari del quadro generale dell'espansione commerciale dei mercanti catalani fra Europa mediterranea e Levante. Si cercherà di raccontare la politica familiare dei Benet integrandola nella società mercantile del tempo.

6 ACB, Llibres extravagants, Comptes de particulars, Libre de compres $i$ vendes di Johan Benet. Càller, 1332-1338. Il registro fu oggetto della tesi di laurea di Maria Marsá Vilá realizzata negli anni '70 del Novecento sotto la direzione di Emilio Sáez, per il Diparitmento di Storia Medievale, facoltà di Geografia e Storia di Barcellona (Marsá, 1977). Fu il primo lavoro sui libri di conti della famiglia Benet. È stato pubblicato uno spoglio del suo lavoro nella rivista Medioevo. Saggi e Rassegne (Oliva, 1980: 65-103). 
In particolare, il lavoro su Johan Benet può contribuire, coi suoi limiti a illustrare la realtà sociale del Castel de Càller del primo Trecento e la sua amministrazione cittadina.

Il libro de deu i deg si è dimostrato ricco di informazioni preziose tra le quali l'elenco completo dei consiglieri della città per il 1335.?

I cinque consellers erano Pere de Vaylmol, Arnau Armangol, Bernat Solzina, Sabrià Siurana, Pere Garau. Si hanno informazioni solo sui primi tre, ricavate da alcuni registri del fondo Real Cancilleria e Real Parimonio dell'Archivio della Corona d'Aragona e dal fondo Antico Archivio Regio dell'Archivio di Stato di Cagliari. Di loro riportiamo soltanto alcuni dati utili alla comprensione dello spessore dei personaggi che vivevano a Cagliari nel primo Trecento e con i quali Johan trattava i suoi affari.

Pere de Vaylmol o Vallmol era un mercante proveniente da Villafranca de Penedés, in Catalogna. Prima di essere lui stesso conseller era stato ambasciatore dei consiglieri presso Alfonso IV nel 1332. Sappiamo, inoltre, che acquistò, come fece anche Johan, un alberch nel carrer dels mercaders da un certo Francesch Planell a cui erano stati assegnati numerosi immobili. ${ }^{8}$ Aveva contribuito alla conquista di Cagliari con la vendita di vino e legname. ${ }^{9}$ Risulta già morto nell'aprile del 1347, sappiamo, infatti, che Miquel ça Rovira, che sarebbe diventato negli anni un uomo d'affari molto famoso a Cagliari, di cui ci rimane il libro di conti pubblicato da Ciro Manca (Manca, 1969), in quell'anno divenne procuratore di suo figlio. Arnau Armangol o Ermengol, fu consigliere di Bonaria già nel 1327, lo fu ancora nel 1335, come risulta dal nostro registro, e nel 1335 venne nominato doganiere dal governatore tenendo l'incarico dal $1^{\circ}$ novembre a tutto aprile del 1336, in modalità provvisoria a causa della morte di Garau Voltor. ${ }^{10}$ Su Bernat Solzina abbiamo pochissime informazioni, sappiamo che apparteneva ad una famiglia di origine maiorchina di mercanti piuttosto facoltosi che contribuirono alla conquista dell'isola con prestiti in denaro all'infante Alfonso.

7 L'elenco dei consiglieri proviene esclusivamente dal Llibre de deu i deg di Johan Benet. ACB, Llibres extravagants, Comptes de particulars, f. 16r. Il dato è stato pubblicato nella rivista RiME (Figus, 2015: 291)

8 Archivio della Corona d'Aragona (da ora in avanti ACA), Cancilleria, Reg. 514, f. 286r-v (1332, dicembre 28).

9 ACA, Cancilleria, Reg. 512, f. 280r (1331, ottobre 25). Vantava ancora crediti da parte dell'amministrazione per il carico venduto.

10 ACA, Real Patrimonio, Reg. 2060, ff. 71r, 97r. 
Inoltre, lo studio della fonte ha aiutato la ricostruzione precisa delle cariche pubbliche della città negli anni 1335-1336.

1335: - Pere Sivader (o Civader), doganiere ${ }^{11}$ di Castel de Càller, ebbe la carica fin dal mese di maggio del 1333. Nel gennaio del $1335 \mathrm{fu}$ sostituito da Garau Voltor; ${ }^{12}$ Riprese l'incarico dalla morte di Voltor fino al novembre dello stesso anno quando fu incaricato Arnau Armangol

- Garau Voltor, doganiere di Castell de Càller, dal mese di gennaio 1335 resse la carica per alcuni mesi, dal momento che sappiamo che morì in un mese imprecisato tra febbraio ed agosto dello stesso anno. ${ }^{13}$ La carica ritornò a Pere Civader fino al novembre del 1335.

- Arnau Armangol, doganiere di Castel de Càller, nominato dal governatore. Tenne l'incarico per sei mesi, dal $1^{\circ}$ novembre del 1335 a tutto aprile del $1336,{ }^{14}$

- Pere Sa Bisbal, ufficiale del Porto ${ }^{15}$ della città; ${ }^{16}$

11 La carica di doganiere, ufficio di nomina regia di tipo patrimoniale, era stata istituita dall'infante Alfonso per la villa di Bonaria nel 1324. Nel 1326 l'ufficio della dogana subì un primo rinnovamento nell'amministrazione che prevedeva l'unità di Bonaria e Cagliari per limitarsi, l'anno successivo, al solo ufficio di Cagliari. Dal 1333 e fino alle successive riforme introdotte da Pietro IV nel 1355 acquista una certa autonomia rispetto agli altri ufficiali periferici fatta eccezione la dipendenza dall'amministratore generale che ne controlla la gestione. Approfondimenti in Olla-Repetto (1969: 22-24), Simbula (2000: 131-134).

12 ACA, Real Patrimonio, reg. 2060, f. 75v. Nel Llibre de deu i deg di Johan Banet, abbiamo 2 registrazioni che testimoniano la sua attività in quei lassi di tempo: f. $4 \mathrm{v}$, «E yo deg-li que em donà per el en Pere Sivader, duaner, disapte XXIX d'uytubrj...50 ll.»; f. 26v, «Deu en Pere Sivader, duaner de Castel de Càler, que li doné disapte IX de satembre l'ayn MCCCXXXV ...11 ll. 5 s.».

13 Ibidem, f. 34v, «Deu en Garau Voltor, duaner, que doné per el an Beneyto Cabot, macip seu, dijous XVIII de janer l'ayn MCCCXXXV...112 ll. 12 s.».

14 ACA, Real Patrimonio, reg. 2060, F. 97v.

15 L'ufficio del portolano, connesso con la dogana e anch'esso di tipo patrimoniale, si occupava della registrazione della contabilità prodotta dalla riscossione delle tasse del porto, della tenuta delle somme riscosse e del successivo versamento al doganiere. Aveva inoltre l'incarico della sorveglianza portuale e delle attività di polizia ad essa connesse. Il Llibre de deu i deg (f. 26v) attesta tale carica come separata da quella del doganiere andando a smentire la convinzione che vedeva, almeno fino al 1338 , i due uffici retti entrambi dal doganiere, dal guardiano del porto o dal bailo; la registrazione porta, infatti, la data di venerdì 23 settembre1335, si veda nota successiva. Olla-Repetto (1969: 24), Alias (2008-2009: 31)

16 ACB, Llibres extravagants, Comptes de particulars, Llibre de deu y deig di Johan Benet 1334-1338, f. 26v: «Item deu que em dix a pagar per en Pere Sa Bisbal, portolà, divenres XXII de satembre l'ayn $M C C C X X X V . . . X X$ lliures». In questa registrazione Pere Civader, doganiere, deve 20 lire a Johan Benet, sottolineando che quei soldi li aveva pagati in vece di Pere Sa Bisbal, portolano, il vero debitore. 
1336: - Arnau Des Torent, doganiere di Castel de Càller, sappiamo che resse la carica per un breve periodo; ${ }^{17}$

- Francesc I Des Corral, fu veguer ${ }^{18}$ dal 29 gennaio al 17 aprile; ${ }^{19}$

- Pere Castany de Viela, fu veguer dal 18 aprile al 19 dicembre; ${ }^{20}$

Ulteriori dati per la conoscenza delle strutture amministrative cagliaritane è venuto dalla nomina, inserita nelle registrazioni, di 7 notai che hanno rogato a Castel de Càller dal 1334 al 1337..1 Se ne ipotizza, tuttavia, un numero superiore dal momento che dovevano servire l'intera città.

La presenza di numerosi pisani operanti in Castel de Càller per quegli anni ha stemperato le affermazioni molto diffuse circa l'espulsione di tutti i pisani dalla città dopo il 1326. Il registro di Johan Benet, infatti, dimostra l'esistenza di 15 presenze tra mercanti e artigiani sia dentro le mura che nelle appendici. ${ }^{22}$

Allo stesso modo troviamo anche un discreto numero di sardi, 14 in questo caso, e quasi tutti commerciavano in tessuti. Abbiamo, inoltre, la presenza di famose compagnie catalane e italiane come i Mitjavila, gli Holivela e i Bardi di Firenze, rappresentate in città dai loro fattori. Numerose sono le cariche istituzionali citate, tutte rette da esponenti dell'alta borghesia catalano-

17 ACA, Real Patrimonio, reg. 2060, f. 97r.

18 La carica di veguer (vicario), di nomina regia, viene istituita nel 1326 dall'infante Alfonso già per la villa di Bonaria passando poi al solo Castel de Càller. Si occupava dell'amministrazione giudiziaria della città e delle appendici come tribunale di prima istanza e talvolta di seconda, in materia sia civile che penale. Dal 1331 estende le sue competenze anche alle ville circostanti Cagliari togliendo potere ai feudatari locali. Aveva anche competenze di natura politica, militare ed amministrativa, arrivando a sostituire lo stesso governatore generale durante le assenze di quest'ultimo dalla città. Rendeva conto del suo operato direttamente al maestro razionale di Barcellona. Olla-Repetto (1969: 26-29).

19 ACA, Real Patrimonio, reg. 2060, f. 82v.

20 Ibidem, f. $93 \mathrm{v}$.

21 Si sottolinea che il numero dei notai è stato considerato tenendo presente i nomi così come l'autore del Llibre de deu i deg li riporta (Ravel, G. Brocoyl, Paul Holiver, Jacme Salent, Galà, Galiyà, P. Galià). A seguito di ulteriori ricerche si può, con ragione, ipotizzare che gli ultimi tre notai indicati tra le parentesi facciano riferimento alla medesima persona abbassando il numero da sette a cinque. I notai più presenti nel registro di Johan Benet sono un certo Ravel e Pere Galià, citati sette volte. Di Ravel sappiamo che operò in maniera ininterrotta dal 1334 al 1337. Le annotazioni riguardano tutte redazioni di carte di comanda per viaggi di mare. Il notaio Pere Galià è citato per gli anni 1335, 1336, 1337. G. Brocoyl e Paul Holiver o Oliver citati nel 1335 per tre volte. Jacme Salent, lo troviamo attestato nel 1335.

22 La presenza dei pisani a Cagliari, anche successivamente alla conquista, è ampiamente documentata in Petrucci (2005-2006: 915-927). Impossibile in questa sede fornire l'elenco di tutte le registrazioni, in questo caso riguardanti i pisani e i sardi, attestate dal registro in quanto costituiscono oggetto della futura tesi dottorale, ci si è limitati, pertanto, a fornirne qualche esempio laddove si è trattato di singoli personaggi o di casi isolati. 
aragonese sui quali sarebbe interessante approfondire, dal veguer ai doganieri, ai consoli dei mercanti.

Un grande spazio occupano i traffici commerciali, non solo quelli tra Barcellona e Castel de Càller ma con Napoli, Trapani, Pisa, Alessandria d'Egitto, la Provenza, Sicilia e la Calabria.

Interessanti sono le citazioni dei prodotti importati da Barcellona, in particolare, i tessuti di molteplici varietà. Altrettanto quelli importati dalla Sardegna, tra tutti, grano e argento.

Quanto agli strumenti finanziari utilizzati nel llibre, ho rilevato una conoscenza e un uso corrente e fluido del cambio delle monete, una discreta presenza della lettera di cambio registrata e usata secondo diverse modalità.

È notevole l'uso della contabilità per definire le comandes commerciali, ${ }^{23}$ così come la citazione piuttosto frequente dei sensali e dei piccoli prestiti personali. ${ }^{24}$

23 La figura della comanda spagnola o commenda, come era chiamata in Italia, come contratto associativo è stata molto presente in tutti gli affari di Johan Benet e il libro de deu i deg ne è un buon esempio. Tra le diverse modalità di comanda emergeva soprattutto la commerciale, vincolata ai viaggi marittimi e della quale nel libro figurano numerosi esempi, generalmente relazionati con grandi distanze.

Ricordiamo che il contratto tipico, di commenda o accomendatio, metteva in relazione un socio finanziatore con un altro viaggiatore, incaricato di rendere effettiva l'operazione commerciale rimessa in un altro luogo. Per i contratti marittimi si veda Tangheroni (1996: 341-350).

24 I prestiti non venivano erogati tutti allo stesso modo e la tipologia di destinatario faceva una grande differenza. Quando venivano concessi a domestici, servitori o gente di assoluta fiducia, tra cui, per esempio, un parente, le somme prestate non superavano mai grandi quantità e non si è trovata traccia di alcun tipo di garanzia come contropartita. Si trattava di un piccolo prestito di carattere domestico, in realtà, piccoli anticipi di salari o di pagamenti di affitti e molte volte la loro componente di credito sarebbe passata completamente inosservata se nelle registrazioni non fosse apparsa la locuzione «li presté». Non si può dire, comunque, che questi prestiti fossero completamente esenti da qualsivoglia tipo d'interesse e l'obbligazione veniva cancellata al momento della restituzione dell'importo. Grazie al fatto che queste annotazioni avevano lo scopo di fungere da luoghi della memoria e che questi piccoli prestiti facevano riferimento generalmente all'oggetto o alla ragione del credito, abbiamo la possibilità di conoscere una serie di informazioni relative al prezzo di molti beni o prodotti. Nei prestiti concessi a personaggi di ceto sociale elevato o di pari livello, ad altri mercanti, artigiani o gente estranea all'ambito domestico, si esigeva un pegno o una garanzia. Questa poteva essere una «carta»o documento notarile che stabiliva la forma contrattuale e il carattere pubblico, tanto per le obbligazioni del prestatore come di colui che riceveva il prestito. Generalmente questi contratti prendevano la forma corrente di una ricevuta attestante il prestito, di carte d'armamento, se avvenivano durante le traversate in mare o delle carte di comanda, ancorché, a volte, per mancanza d'informazioni complementari o per la brevità della stessa registrazione (o registrazioni), potremo arrivare a confonderle con comandes strettamente commerciali o comandes con una forte connotazione monetaria o finanziaria. Nonostante ciò, alcune di queste carte di comanda registravano operazioni di prestiti, molte volte importanti e nelle quali non vi era alcuna componente commerciale. Altre volte non era necessario passare dal notaio e una semplice ricevuta (albarà) era sufficiente come forma di fiducia o garanzia. Abbiamo anche casi 
Senza dubbio, la presenza più significativa è quella della dita, una sorta di formula pratica che permetteva ordini di pagamento pienamente eseguibili e non necessariamente vincolati al mondo bancario (Melis, 1972: 84-85). Anche nel llibre de deu $i$ deg, senza ignorare quelle dite che, nel libro, realmente documentavano un credito o nascondevano una situazione di prestito, la maggior parte delle volte le dobbiamo considerare come un ordine di pagamento. Alcune volte, gli ordini non richiedevano nessuna nota speciale o carta scritta e si davano oralmente, sia davanti ad una tavola di cambio e in presenza di un cambista o semplicemente tra gli attori principali dell'operazione finanziaria, nella bottega, in casa o in qualsivoglia altro luogo. ${ }^{25}$ Johan Benet e i mercanti/ cambisti che si muovevano nel suo giro d'affari, mantenevano tutta una rete finanziaria basata sul meccanismo della dita, la quale permetteva di dare molta fluidità ai loro affari. Nel llibre, la dita appare secondo diverse forme grammaticali, relazionate con il «dix» o coniugazione del verbo dir alla terza persona singolare, indicando in questo modo che i protagonisti erano sempre il titolare del conto o Johan Benet, ossia, i due attori principali che intervenivano nei trasferimenti di denaro, in una posizione debitoria o creditizia. ${ }^{26}$

Tale strumento finanziario si rivela ricco di numerose sfaccettature, per cui dare in questo frangente una spiegazione esauriente della dita richiederebbe uno spazio troppo lungo. $\mathrm{Ci}$ si riserva di affrontare la tematica in maniera approfondita nella stesura della tesi dottorale. ${ }^{27}$

Associati a questi aspetti ci sono anche quelli legati alla quotidianità e alla religiosità. Troviamo infatti notizie interessanti sulle elemosine fatte ai conventi della città, in particolare ai frati predicatori della chiesa di Santa Eulalia e ai frati minori; le offerte per le messe nella chiesa di Bonaria, di cui si rileva la prima attestazione della sua esistenza; ${ }^{28}$ le malattie più diffuse e le loro cure;

per cui si esigeva un pegno o una garanzia che si materializzava in denaro, gioielli o casse d'argento. De Roover (1948); Madurell-Sanz (1973: 204-205).

25 ACB, Llibres extravagants, Comptes de particulars, Llibre de deu y deig de Johan Benet 1334-1338, f. 28r, Tomàs Saguí deve a Joahn Benet 4 11. 1 s.. In questa registrazione l'ordine di pagamento delle spese del vitto vengono date oralmente a casa di Pere Adrover: «Item deu que dixí a pagar per el an Pasqual Des Col per mació de manjar que el dit Tomàs avia fet en caza d'en P. Adrover per LIIII jorns...4 ll. 1 s.».

26 ACB, Llibres extravagants, Comptes de particulars, Llibre de deu y deig de Joan Benet 1334-1338, f. 2v, in un conto chiuso a nome di Pons Raholench abbiamo: «Deu en Pons Raholench que em [me, Johan Benet] dix ha pagar per Bero et per Meylo...». Pons Raholench paga Johan Benet per conto di Bero e Meylo.

27 Sulle origini della dita in Catalogna, García- Sanz (1964-67: 81-87), Conde (1988: 128).

28 ACB, Llibres extravagants, Comptes de particulars, Llibre de deu y deig di Johan Benet 1334-1338, f. 9v: «Item deu que doné an Bernat Claret a I mi/sa que fou dir a Bonayre per la fi/la d'en Berenguer Banet...I sous VI». 


\section{Simonetta Figus}

l'acquisto di alberchs, ${ }^{29}$ l'affitto di botteghe, gli acquisti a titolo personale di prodotti od oggetti di lusso, come un capello da sole, che Johan Benet si fece arrivare da Napoli, i giochi in uso in città, una registrazione, infatti, testimonia l'uso (in alcuni casi l'abuso) di un gioco d'azzardo chiamato taules molto diffuso in Catalogna, tanto che alla fine del secolo XIv a Barcellona venne proibito, ${ }^{30}$ i pranzi e le cene.

Sono molti gli aspetti cui il registro accenna ma non rende possibile, almeno per il momento, effettuare approfondimenti, come i rapporti creditizi o debitori col clero, con gli ebrei, o con certi personaggi conosciuti e in vista tra la nobiltà catalana, come Ramon de Vilaragut, priore di Santa Maria di Montserrat, figlio di Guillem, governatore di Xativa, incontrato attraverso il suo procuratore Pere de Raxach. ${ }^{31}$

\section{Prodotti provenienti da Cagliari}

Il registro mostra alcuni dei prodotti che maggiormente si esportavano fuori dall'isola e che sono perfettamente in linea con quanto già si conosceva sull'argomento $^{32}$ relativamente ai secoli XIII-XIV. La produzione agraria nell'isola, che insieme all'estrazione del sale, costituiva la maggiore fonte di export che la Sardegna potesse vantare, sebbene non l'unica, ricordiamo infatti l'argento, la lana e il cuoio, il vino e i formaggi tanto per fare un piccolo esempio, è un aspetto ampiamente documentato nel registro di Johan Benet e altrettanto nel registro studiato in precedenza da Maria Marsà, appartenente anch'esso a Johan Benet e di cui si è già trattato.

Nel passare prima in rassegna i vari prodotti trattati dal nostro mercante è opportuno soffermarsi un poco sul commercio del grano, vuoi per la sua forte

29 ACB, Llibres extravagants, Comptes de particulars, Llibre de deu y deig di Johan Benet 1334-1338, f. 32v: «E yo deg-li per un alberch que compré / de el per mà d'en Bernat Casteyló, corador de col, diemenge XXVI de noembre l'ayn MCCCXXXV a encant póblic / per preu de CV lliures».

30 Gioco simile alla dama, nel quale si usava una tavoletta divisa in due parti e ciascuna di queste in dodici caselle quadrate alternativamente bianche e nere, dentro le quali i giocatori posizionavano i loro dischetti di legno, affidando la sorte ai dadi; vinceva chi per primo riusciva a posizionarle secondo un ordine prestabilito.

31 ACB, Llibres extravagants, Comptes de particulars, Llibre de deu y deig di Johan Benet 1334-1338, f. 59r: «Deu en P. de Raxach, procurador del prior de Monserat, que doné per el a l'ascrivà de l[a nau] d'en Olivela XXV barchelonezes, qui [valen]...[...]».

32 A tale proposito si vedano Artizzu (1961-62: 14-21 e 1958), F. Melis (1972: 104-119), relativamente alla fabbricazione e al commercio dei prodotti tessili. C. Manca (1966: 114-117). 
presenza all'interno del registro, vuoi perché ci sembra che non si possa fare a meno di rilevare, almeno ancora per tutta la prima metà del Trecento, un certo "benessere" nella produzione di cereali in Sardegna. Ciò nonostante, è opinione comune che nel Trecento le difficoltà dell'approvvigionamento granario crebbero e si generalizzarono (Tangheroni, 1981:14). Senza soffermarci sulle cause di queste difficoltà, si può qui rilevare che i dati contenuti nel registro di Johan Benet assumono, a tal proposito, ancora più valore ed importanza dal momento che nella seconda metà del Trecento, come ci conferma Marco Tangheroni, si assiste, alla scomparsa o quasi del grano sardo dal mercato (Tangheroni, 1981:18).

I cereali: Già in epoca pisana, dallo studio dei registri doganali delle merci varie, Tangheroni ha potuto registrare una rilevante presenza di mercanti e operatori economici provenienti da tutti i centri del Mediterraneo occidentale e un volume d'affari interessante per l'epoca (Tangheroni, 1973: 119-126), «in un intreccio di rapporti mercantili, armatoriali, dei più complessi» (Tangheroni, 1981: 62-63), avvantaggiati dall'assenza del predominio catalano nel commercio sardo.

I primi ventisei anni di dominio catalano nell'isola videro la situazione economica pressoché immutata rispetto al periodo pisano, nonostante la peste del 1348 che secondo John Day avrebbe contato il 40\% di morti nella popolazione rurale (Day, 1978:156). Dopo il 1354, l'economia sarda voltò pagina in conseguenza dei danni particolarmente sentiti e profondi che la guerra contro l'Arborea lascerà sia in termini di produttività che di vite umane.

Già durante la conquista della Sardegna le truppe catalane dovettero affrontare diversi momenti di difficoltà nell'assicurarsi le vettovaglie necessarie, specie quelle cerealicole, sia sotto forma di gallette o biscotti, sia in frumento e orzo (Arribas-Palau, 1952: 189 e segg.). Tale difficoltà sussistette anche negli anni successivi, nel registro sono, infatti, presenti varie registrazioni di commercio di biscotti, ${ }^{33}$ importanti per chi andasse per mare e il fatto che a Castel de Càller una delle vie fosse intitolata ai biscuyters, la dice lunga sull'importanza che questa categoria rappresentava nel panorama alimentare di una città portuale. Tale situazione ebbe il suo miglioramento dopo la definitiva vittoria dei catalani sui pisani nel 1326 e grazie ai rapporti ancora

33 ACB, Llibres extravagants, Comptes de particulars, Llibre de deu y deig, f. 13v, conto di B. Des Grau, biscottaio: «Deu en B. Des Grau, bascuyter, que li he prestat entre II vegades...3 ll.»; Acquisto di biscotti per 36 quintars, 33 libbre, 4 once dallo stesso des Grau, al prezzo di 2 s. 8 dr. Per quintar: «E yo deg-li per faedures de XXXVI quintars XXXIII lliures IIII onzes de bascuyt, que em fou a rahó de II sous VIII diners lo quintar; munten...4 11. 17 s.». 
buoni che la Corona manteneva coi giudici d'Arborea. Nel registro sono presenti diversi rifornimenti di frumento fatti ad Oristano ${ }^{34}$ per un totale di 2661 starelli equivalenti a 130.921, 2 lt. ${ }^{35}$ Ai fini delle osservazioni fatte in precedenza, a proposito delle condizioni commerciali rimaste inalterate nei primi anni di dominio, è importante ricordare come uno dei carichi, di proprietà di Johan Benet ma realizzato dal pisano Andrea Gambarino, 108 starelli, avvenne su una nave pisana, quella di Neri de Xorce, scaricato a Porto Pisano e venduto nella piazza del grano dove fruttò $3111.8 \mathrm{dr}^{36}$

Figura 1. Tabella riassuntiva sulle quantità e i relativi prezzi del frumento comprato ad Oristano

\begin{tabular}{|c|c|c|}
\hline Starelli & Prezzo percentuale* & Prezzo totale di vendita \\
\hline 108 & $23 £$. & $24 £ .16 \mathrm{s.} 9 \mathrm{dr}$. \\
\hline 108 & & $31 £ .8 \mathrm{dr}$. \\
\hline $833+1 / 3$ & & $244 £ . * *$ \\
\hline 1612 & & $128 £ .1 \mathrm{s.} 6 \mathrm{dr} . * * *$ \\
\hline
\end{tabular}

* prezzo calcolato su una base di 100 starelli.

** totale compreso di spese accessorie.

*** prezzo calcolato sulla terza parte spettante al Benet.

A questa interessante registrazione se ne aggiungono altre quattro, di grande valore, che fanno riferimento alla guerra tra l'Aragona e Genova (13301336). La prima registrazione si trova nel "deu" del conto di Bernat Sa Pila. Il predetto debitore deve pagare Francesch De Palau, armatore catalano, poiché si trovava momentaneamente sprovvisto di denaro, Johan Benet paga il De Palau per conto di Sa Pila la cifra di 8 1l. e $8 \mathrm{~s}$ che sono ciò che gli spettava del riscatto pagato dai genovesi per le 400 salme di cereali che erano custodite, con gl'interessi, nella nave di Sa Pila.

34 Ibidem, f. 54v, registrazione di un carico di frumento che Johan Benet ha comprato ad Oristano: «Item li deg per DCCCXXXIII starels e ters de forment que comprà per mi en Horistany en lo mes de daembre l'ayn MCCCXXXVI, los quals costaren per tots, fetes macions...244ll. 12 s. 9dr.»; f. 80r, carico di frumento comprato e inviato a Barcellona da Oristano: «Item li deg per lo ters de so que costaren MDCXII starels de forment que careguà en la coqua d'en Almenare en Horistany...128 ll. $1 \mathrm{~s}$. $6 d r . »$

35 Sulla capacità dello starello di Cagliari, equivalente a 49,2 litri, si veda Manca (1966: 335).

36 ACB, Llibres extravagants, Comptes de particulars, Llibre de deu y deig di Johan Benet 1334-1338, f. $24 \mathrm{v}$, « Deu Andrea Gambarino per CVIII starels de forment que caragà per mi en Horistany en lo leny de Ner de Xorce, pizà, diluns a X de mag l'ayn MCCCXXXV, lo qual anà en Piza descaragat; e ha-se'n en Piza haut...31 11. 8 dr.» 
Evidentemente, quel carico di cereali era di notevole importanza per i genovesi, ma lo era anche per i catalani per i quali erano venuti meno, a causa della guerra, i rifornimenti con la Sicilia. A queste salme se ne aggiungono altre 200, poi altre 1090 e infine altre 50, per un totale di 1740 salme che equivalgono a 483.720 litri di cereali.

Figura 2. Tabella riassuntiva sulle quantità e i relativi prezzi del frumento riscattato dai genovesi

\begin{tabular}{|c|c|c|}
\hline Salme & Prezzo percentuale* $^{*}$ & Prezzo totale di vendita \\
\hline 400 & & $8 £ .8 \mathrm{~s}$. \\
\hline 200 & & $4 £ .4 \mathrm{~s}$. \\
\hline 1090 & $2 £ .2 \mathrm{s.}^{*}$ & $22 £ .17 \mathrm{~s} .9 \mathrm{dr}$. \\
\hline 50 & & $1 £ .1 \mathrm{~s}$. \\
\hline
\end{tabular}

* prezzo per 100 salme.

Non soltanto cereali, però. Da altre due registrazioni, sempre appartenenti al conto di Bernat Sa Pila, veniamo a sapere che il sequestro non si limitò ai soli cereali ma si estese a uomini e navi genovesi per riavere i quali questi ultimi furono costretti a pagare un riscatto. Nella prima, segnata nella parte del "deg", Johan Benet annota di dover pagare 14811.18 s. 4 dr. al Sa Pila poiché Johan Nabot, un intermediario, ha dato al Benet da parte di Sa Pila il denaro pagato dai genovesi per il riscatto della loro nave e dei marinai $;{ }^{37}$ nella seconda, troviamo gli stessi protagonisti ma, in questo caso, il riscatto viene pagato per liberare il capitano delle navi genovesi caduto nelle mani dei catalani. Johan Benet viene pagato dal Nabot per la ventesima parte del riscatto che dovrà poi essere restituita al Sa Pila, corrispondente a 38 1l. 4 s. 2 dr. ${ }^{38}$ Di tutte queste registrazioni riguardanti i genovesi non troviamo nessuna indicazione di carattere temporale.

Le condizioni favorevoli degli anni immediatamente successivi alla conquista crearono un traffico frequente di esportazioni di cereali tra la Sardegna e Barcellona e, poiché la situazione in Catalogna non aveva ancora

37 ACB, Llibres extravagants, Comptes de particulars, Llibre de deu y deig di Johan Benet 1334-1338, f. $5 \mathrm{v}$, «E yo deg-li que em donà per el en Johan Nabot per lo rescat dels jenovezos, d'asò qui en vania a la nau ab los mariners...148 ll. $18 \mathrm{~s} .4 \mathrm{dr} . »$

38 Ibidem, f. 5v, «Item li deg que em donà lo dit Johan Nabot per lo vinté del capità de les naus...38 ll. 4 s. $2 d r . »$ 
assunto i caratteri di drammaticità che acquisterà in maniera particolare dopo il 1333 a causa della carestia che la colpì, ancora era possibile trovare esportazioni verso altri porti del Mediterraneo. Tuttavia, dall'estate del 1332 una nuova politica economica venne a condizionare i traffici in Sardegna. Infatti, secondo alcuni documenti editi da Boscolo, se i mercanti catalani volevano esportare il grano sardo avevano bisogno di un ordine reale e anche questo era comunque soggetto alla valutazione del governatore locale (Boscolo, 1973, docc. n. 240, n. 239). Alla fine dello stesso anno un provvedimento del governatore vietò l'esportazione dei cereali in tutti quei paesi che non appartenessero alla Corona o al regno di Maiorca. ${ }^{39}$ Ma nel 1335 fu revocato con la motivazione che l'amministrazione aragonese della Sardegna aveva necessità di coprire il deficit che si era creato incrementando le vendite di cereali. $^{40}$

In realtà ci sembra opportuno considerare, a proposito delle entrate doganali sarde, l'incidenza sia della treta del forment e de l'ordi che dell'ajuda che nel complesso appaiono assai elevate, considerando gli anni dal 1334 al 1337 si rileva dal registro di Johan Benet, un'entrata complessiva di 203411.14 s. 11 dr. (naturalmente di alfonsini minuti): di queste erano dovute per la treta dei cereali 86711.18 s. Considerando che il diritto di treta era di $18 \mathrm{dr}$. per starello di frumento e di 12 per l'orzo, si può avere un'idea approssimativa del volume delle esportazioni. L'idea di queste cifre non può, in ogni caso, che essere approssimativa poiché legata alle registrazioni non sempre affidabili, spesso scarne d'informazioni o più spesso legate a calcoli personali del nostro mercante. Nonostante la proporzione tra grano ed orzo non fosse affatto analoga ma vedesse tra i due cereali un notevole stacco che favoriva il frumento, si può calcolare un'esportazione complessiva tra il 1334 e il 1337 di 48.000 starelli circa, pari a circa 24.000 ettolitri. A cui vanno opportunamente aggiunte le registrazioni delle salme di cereali, frumento e orzo, per complessive 3.602 salme pari a circa 10.000 ettolitri.

Il 1333 rappresentò un anno assai difficile per la Corona, Alfonso IV il Benigno informò il governatore Ramon de Cardona che a causa della carestia che stava mettendo in ginocchio la Catalogna sarebbero giunte in Sardegna alcune cocche ed altre imbarcazioni per rifornirsi di grano (Boscolo, 1973, docc. n. 337). Per l'appunto, la situazione non era facile neanche in Sardegna. 
Bernat Des Col (o Dez Coll, Des Coll) addetto per la corte alle questioni finanziarie ed economiche sarde e presente in due registrazioni del nostro llibre, ${ }^{41}$ stende nel maggio del ' 33 un rapporto in cui descrive la grave situazione sarda con particolare riferimento alla cerealicoltura e ai suoi problemi (Tangheroni, 1981:86-87). A suo parere, la situazione produttiva nell'isola era del tutto carente e incapace di far fronte ai reali bisogni della Corona. Nel 1334 la situazione migliorò per quanto riguardava i raccolti che furono capaci di creare un surplus anche se, probabilmente, ciò si rese possibile soltanto grazie al sacrificio dei consumi locali. Tuttavia, la situazione andò migliorando con gli anni, all'inizio del regno di Pietro IV si poté registrare un periodo di ripresa delle potenzialità esportatrici dell'isola.

Attraverso il libro di conti del doganiere di Cagliari Pere Civader, la cui attività per l'anno 1335 è testimoniata anche nel nostro llibre, sappiamo che le entrate del dret della dogana, per un periodo che si aggira al 1335, erano state, in totale, 196811.2 s. 11 dr.; nel registro di Johan Benet, per gli anni 1335-1337, ne abbiamo potuto calcolare 116611.20 s. 11 dr., cifra che, ci pare, abbastanza affidabile se diluita in un triennio. Le entrate della treta del frumento e dell'orzo, sempre intorno al 1335, ammontavano a 549311.11 s. 6 dr. Dal nostro registro, per lo stesso triennio considerato poc'anzi, abbiamo potuto rilevare la cifra di $86711.18 \mathrm{~s}$. Vi fu però un evento di grande importanza che cambierà il senso di queste cifre, ossia la decisione di appaltare le entrate doganali dell'ajuda ad alcuni uomini d'affari catalani, tra cui, per il 1335, Johan Benet. L'appalto gli verrà concesso domenica 9 aprile 1335 per 1.300 1l.; ne entrò ufficialmente in possesso mercoledì 12 aprile, per un anno.

Detto questo, stabilire il prezzo del grano e dell'orzo con precisione per annualità è quanto mai difficile per tutte quelle carenze a cui si è più volte accennato, la mancanza di una datazione precisa e costante, l'assenza di informazioni aggiuntive e incognite mancanti perché date per scontate dal nostro mercante. Ciononostante, possiamo azzardare alcuni dati relativi ai prezzi per anno e prodotto che è stato possibile stabilire con certezza, nello specchietto qui, di seguito:

41 ACB, Llibres extravagants, Comptes de particulars, Llibre de deu y deig de Joan Benet 1334-1338, f. $5 \mathrm{v}$, in questa registrazione Bernat des Col appare avere una tavola dei cambi presso cui il Benet e Bernat sa Pila si recano per un cambio di valuta: «Item li deg que em donà en Beneyto Ferrer, los quals el avia rabuts més del cambi que fem el e yo ab en Bernat Des Col...10 s.»; f. 52 v, qui, il doganiere Arnau des Torent paga Benet al posto di Bernat des Col per un carico di stoffe: «Deu n'Arnau Des Torent, duaner, que em dix ha pagar per en Bernat Des Col dimecres XI de daembre per I baló d'estopes, qui bastà ha CCCLVIII canes e mig, qui a rahó de X lliures V sous lo centenar munten...36 ll. $14 \mathrm{~s} .11 \mathrm{dr} . »$ 


\begin{tabular}{|c|l|c|}
\hline Annualità & \multicolumn{1}{|c|}{ Frumento* $^{*}$ Orzo* $^{*}$} \\
\hline 1334 & $45-46 £$. & $20 £$. \\
\hline 1335 & $34 £$. & $10-12 £ .12$ s. 3 dr. \\
\hline 1336 & da 21 a $32 £ .12$ s. 9 dr. ** & $14 £ .15$ s. 3 dr. \\
\hline 1337 & $36 £ .12$ s. 8 dr. & \\
\hline
\end{tabular}

* Prezzo di 100 starelli.

** Prezzo del grano rilevato nel dicembre del 1336 nella piazza di Oristano.

Lo studio dei prezzi di tali prodotti è sempre impreciso e ancora di più lo è per quanto riguarda i cereali, perché di tutti i beni oggetto di traffici i cereali sono quelli più soggetti alle variazioni annuali e stagionali e alla qualità che in taluni casi poteva fare la differenza e influenzare le vendite. Nella tabella che presentiamo qui sotto troveremo alcune delle oscillazioni di prezzo relative a frumento ed orzo rilevate dal registro di Johan Benet; noteremo la varietà di prezzo con picchi anche piuttosto elevati. Tra questi prezzi non è possibile documentare quali siano praticati al minuto e quali all'ingrosso, quali siano al netto e quali al lordo; i prezzi considerati sono relativi agli anni 1334-1337:

\begin{tabular}{|c|c|}
\hline & Prezzo orzo \\
\hline \multirow[t]{2}{*}{ Prezzi per 1 starello } & $2 \mathrm{~s} 10 \mathrm{~d}$ \\
\hline & $4 \mathrm{~s}$ \\
\hline \multirow[t]{5}{*}{ Prezzi per 100 starelli } & $10 £ .12 \mathrm{~s}$ \\
\hline & $12 £ .11 \mathrm{~s} 3 \mathrm{~d}$ \\
\hline & $14 £ .5 \mathrm{~s}$ \\
\hline & $20 £ .1 \mathrm{~s} 5 \mathrm{~d}$ \\
\hline & Prezzo frumento \\
\hline \multirow[t]{5}{*}{ Prezzi per 1starello } & $5 \mathrm{~s}$ \\
\hline & $5 \mathrm{~s} 6 \mathrm{~d}$ \\
\hline & $5 \mathrm{~s} 7 \mathrm{~d}$ \\
\hline & $7 \mathrm{~s}$ \\
\hline & $9 \mathrm{~s}$ \\
\hline
\end{tabular}




\begin{tabular}{|l|l|}
\hline Prezzi per 100 starelli & Prezzo frumento \\
\hline & $18 £ .10 \mathrm{~s}$ \\
\hline $23 £$. \\
\hline $24 £ .10 \mathrm{~s} 8 \mathrm{~d}$ \\
\hline $27 £$. \\
\hline $29 £ .10 \mathrm{~s}$ \\
\hline $30 £ .10 \mathrm{~s}$ \\
\hline $31 £$. \\
\hline $31 £ .6 \mathrm{~s} 8 \mathrm{~d}$ \\
\hline $32 £$. \\
\hline $32 £ .9 \mathrm{~s}$ \\
\hline $33 £$. \\
\hline $33 £ .10 \mathrm{~s}$ \\
\hline $33 £ .5 \mathrm{~s}$ \\
\hline $35 £ .1 \mathrm{~s} 7 \mathrm{~d}$ \\
\hline $36 £$. \\
\hline $36 £ .10 \mathrm{~s}$ \\
\hline $36 £ .16 \mathrm{~s} 8 \mathrm{~d}$ \\
\hline $37 £$. \\
\hline $45 £$. \\
\hline $46 £$. \\
\hline
\end{tabular}

\section{Prodotti provenienti da Barcellona}

Il registro de deu i deg di Johan Benet fornisce una gran varietà di prodotti commercializzati nella piazza di Castel de Càller durante la prima metà del Trecento. Nel registro sono citati una cinquantina di prodotti e il loro relativo prezzo, sia quello che veniva effettivamente pagato sulla base della quantità sia il costo singolo. I prodotti sono stati considerati non solo per la loro provenienza ma anche, e soprattutto, per il loro prezzo di vendita e per quello con cui viene immesso nel mercato, dato di grande importanza per una conoscenza più 
precisa sui dati economici delle piazze commerciali del Mediterraneo Occidentale nel primo Trecento. Abbiamo voluto trattare in questo breve saggio anche i prodotti provenienti da Barcellona in Sardegna.

La frequenza con cui un prodotto viene citato nei singoli conti ci dà la misura del suo uso o consumo, dell'oscillazione del prezzo negli anni considerati dal registro e l'affidabilità dei dati forniti proprio dalla sua ripetitività.

A tale proposito, il prodotto proveniente da Barcellona che ottiene il primato tra le registrazioni è, senz'altro, il tessuto, inteso nelle sue molteplici varietà.

Se ne fornirà un elenco seguendo un ordine alfabetico:

Barragans: (in italiano barracani) sono citati otto volte, nelle forme barragans, baragans di piccolo formato e di formato medio. Secondo la "Nomenclatura de los tejidos españoles del siglo XIII" (da ora Nomenclatura) di Alfan de Solalinde, si trattava di un tessuto a righe da principio in lana e successivamente in seta. Il dizionario dell'Alcover-Moll ${ }^{42}$ (Alcover-Moll, 193062), dice che era un tessuto di lana impermeabile, molto usato nelle navi per mitigare le luci, simile ad un pezzo di coperta. Il prezzo di vendita riportato in due registrazioni datate, una a giugno e l'altra a dicembre 1335, ci ha permesso di rilevare il forte aumento avvenuto nel giro di appena sei mesi. Il registro non specifica se la differenza di prezzo sia dovuta a differenti qualità di tessuto, possiamo soltanto rilevarne l'innalzamento e affermare che la registrazione cita semplicemente barragans, senza ulteriori aggiunte, in entrambi i casi; a giugno il prezzo per 1 pezza di barragans era di 17 s., a dicembre il prezzo sale a $22 \mathrm{~s}$. per pezza. Nel 1336 il prezzo per barragans mediani ammonta a $21 \mathrm{~s}$. per pezza. Nel 1337 il tessuto costa 18 s. 4 dr. la pezza, i barragans piccoli 15 s. e quelli mediani $18 \mathrm{~s}$. la pezza. È molto interessante notare che il prezzo di vendita a Barcellona, ci dice Maria Marsà, nell'aprile del 1335, risulta essere di 10 s. 6 dr. per pezza, notevolmente inferiore rispetto a quello praticato a Cagliari (Marsà Vila, 1977: 55). La differenza è giustificata dalle spese di trasporto e dalle tasse gravanti sui prodotti che inevitabilmente fanno salire i prezzi e, ovviamente, dal guadagno che deve avanzare.

Cadins: sono citati per cinque volte, nelle varietà di cadins blanch e bruns. Secondo l'Alcover, si tratta di un tessuto di lana lavorato ad imitazione dei tessuti inglesi, trasparenti e leggeri ma resistenti. Nel 1337 una canna di cadins blanch costava $4 \mathrm{~s} .10 \mathrm{dr}$. Una cana equivaleva a 8 palms. Il palm o pam era la 
Figura 3. Diagramma che espone i prezzi dei Barragans classificati per anno. Al centro troviamo il valore più alto

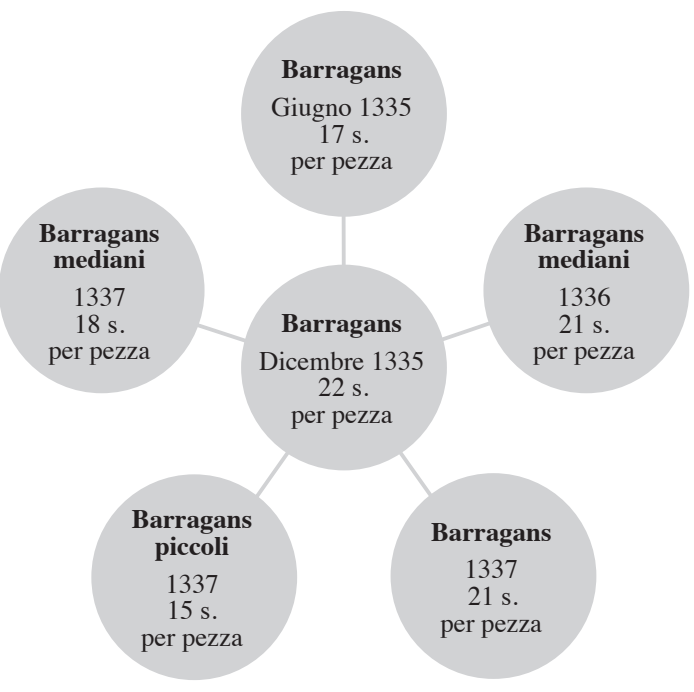

misura corrispondente ad una mano, ossia alla distanza che normalmente intercorre tra il pollice e il mignolo, tenendo la mano aperta e le dita distese (Alsina-Feliu-Marquet, 1990: cana 130-131, palm/pam 188-189).

Canabàs: è citato 22 volte nelle registrazioni del llibre. Si trattava di una tela fatta dalla pianta del cànem della famiglia delle canabàcies. Nel $1333 \mathrm{a}$ Barcellona il prezzo era di 2 11. 18 s. per 100 canne, nel 13374 11. 4 s. A Castel de Càller nel 1334 aveva un prezzo di 17 dr. per canna, nel 1335 costava 16 dr., nel 1336 il prezzo aumenta leggermente di un denaro salendo a 18 dr. per canna; non abbiamo dati certi per il 1337-38. Molte delle registrazioni di vendita di canabàs non ci forniscono date.

Drap: si riscontrano registrazioni per 88 partite di drap; in italiano drappo si riferisce ad una stoffa di lana morbida, lucida, setosa. Il dizionario dell'Alcover dice che si trattava di roba o tessuto di qualsivoglia specie, poteva servire per fare abiti, lenzuola, fazzoletti e roba di varia qualità. L'enciclopèdia catalana dice che si trattava di un tessuto di lana, filo, seta o cotone, quando drap si trova espresso senza ulteriori specificazioni allora si allude ad un tessuto di lana (Gran Enciclopèdia Catalana, 2000). Secondo la normativa stabilita dall'associazione del gremio dei tessitori barcellonesi dal 1317 al 1387 , una pezza di panno doveva avere una lunghezza di 20 canne che poteva variare 
solamente in base alla qualità del tessuto (Riu, 1964: 548-549). Inoltre, i mercanti potevano misurarlo soltanto con la canna di Barcellona (equivaleva a 8 palmi o a 1,50 m. circa) e non potevano usare altro sistema di misura anche se si trovavano lontano dalla città comitale (Pinna, 1928: ordinazione n. 30).

Si ritrovano citati molteplici tipologie di drap tra cui:

Drap de Pugcerdà. Si trova citato 33 volte, compreso il tipo blaus (1), il tipo comuns (3), il drap mijans (medio, 4) ${ }^{43}$ Era un tessuto tipico della zona della bassa Cerdanya, in Catalogna dove si trovavano alcune manifatture tessili fin dal 1270. A Barcellona il prezzo oscillava tra le 511.10 s. e le 7 11. la pezza. A Cagliari a marzo del 1335 il suo prezzo era di 12 1l. la pezza, nel 1336 il prezzo scende a $1111.10 \mathrm{~s}$. la pezza, a gennaio del $13371211.10 \mathrm{~s}$.

Drap de Perpenyà: è citato una sola volta. Tessuto prezioso proveniente da Perpignano, Nella piazza cagliaritana 10 pezze avevano un prezzo di 175 11. 6 s., un prezzo chiaramente elevato dato dall'alta qualità del tessuto.

Drap de Vilafranca de Conflent: lo si trova citato due volte, era un tessuto di qualità. Anche Vilafranca, che oggi si trova in territorio francese, nel medioevo faceva parte dell'alta Catalogna, nei Pirenei orientali. Non abbiamo anno di riferimento in nessuna delle due citazioni ma sappiamo, dal conto di P. Baraler, ${ }^{44}$ che per 24 pezze sono state pagate $31511.15 \mathrm{~s}$.

Drap de Banyoles: è citato una sola volta. Tessuto proveniente dalla cittadina di Banyoles in provincia di Girona. Sappiamo che, sempre dal conto di P. Baraler, nella piazza di Cagliari, 10 pezze sono costate $2111.4 \mathrm{~s}$. Secondo i dati forniti dalla Marsà, a Barcellona nel 1332 il prezzo era di 4 11. e 10 s. per pezza (Marsà Vila, 1977: 58) per cui, stando a questo dato, per 10 pezze si sarebbero dovute pagare $211.2 \mathrm{~s} .5 \mathrm{dr}$ a pezza.

Drap de Berga: citato una sola volta. Tessuto proveniente dalla città di Berga in Catalogna a nord di Barcellona, anch'essa vicina ai Pirenei. L'incasso, risalente a luglio del 1337, per una partita di 6 pezze è di $6611.12 \mathrm{s.} 9 \mathrm{dr}$.

Drap de li: citato nove volte. Secondo Miguel Gual, proviene da Vich, in Catalogna, famosa per la produzione di tessuti di lino. Ma due registrazioni lo fanno provenire da Alessandria d'Egitto. Tutte le misurazioni sono effettuate

43 I numeri indicati tra le parentesi fanno riferimento alle citazioni riscontrate nel registro.

44 ACB, Llibres extravagants, Comptes de particulars, Llibre de deu y deig di Johan Benet 1334-1338, f.79r.: «Item deu que ha haut de XХIIII peses de draps de Vilafranqua de Conflent, que raebé per la nau d'en Pug he d'en Niubó, de què ach CCCXV lliures XV sous, de què en vé a mi per la tersa part... CV lliures V sous» 
per centinaio di canna, non per pezza come nel caso degli altri draps. A Barcellona, il prezzo più basso (1333) è di 411.6 s., mentre quello più alto è di 7 1l. 1 s. per il 1337 (Marsà Vila, 1977: 59). A Cagliari, nel 1334, il prezzo è di 2 s. 3 dr per canna, ancora più basso di quello registrato a Barcellona nel 1333, nel giugno del 1335 sale a 12 s. per canna.

Drap de Limós: è citato dodici volte. Ben undici nella qualità di drappi misti, ossia tessuti con fili di qualità diversa. Una partita solamente è di drappo colorato. Panno fabbricato nella città di Limoges, nella Francia centrooccidentale, a sud di Parigi, conosciuta nel medioevo per i suoi blu, bruni, rossi, bianchi e verdi de Limós tutti nominati nella prima metà del Trecento. Il drappo misto, a Barcellona, aveva un prezzo di $811.10 \mathrm{~s}$. A Cagliari, nel novembre del 1336, di 16 1l. la pezza, un rincaro di circa 811 tra Barcellona e Cagliari.

Drap de palmeles de Carcasona: è citato cinque volte. Si tratta di un tipo di tessuto di tela di differenti toni di azzurro, originario di Carcassonne, città francese della Linguadoca-Rossiglione. Viene venduto a Barcellona ad un prezzo che oscilla tra le 10 1l. per pezza nel febbraio del 1336 a $1211.28 \mathrm{~s}$. nell'ottobre del 1337. A Cagliari nel marzo del 1335 viene venduto a $241110 \mathrm{~s}$. la pezza, nel 1336 a $2311.13 \mathrm{~s}$. la pezza.

Drap de Toloza: è citato due volte. Nelle qualità mista e a scacchi, in quest'ultima versione, infatti, si aveva una stoffa disegnata a quadri, simile al disegno di una scacchiera. Proveniva da Tolosa, città della Francia, in Occitania. Non si hanno dati circa il prezzo di vendita.

Drap de Bruges: citato tre volte. Una delle registrazioni parla di drap de Bruges aranjat, ossia arancione. Questo tessuto proveniva dalla città di Bruges, nella Fiandra occidentale ed era considerato un prodotto di lusso. La fortuna della città risiedeva nel diretto controllo sul commercio dei tessuti nell'Europa del nord, insieme alle due città rivali di Ypres e Gand, trasformando in particolare la lana inglese di alta qualità in vestiario e tessuti che erano esportati in tutto il mondo conosciuto di allora. Infatti, come ci spiega il Madurell, a Bruges vi era una colonia di mercanti castigliani fin dal secolo XIII che comprava tessuti e li importava nella penisola iberica (Madurell - Garciá, 1973: 107-114). Veniva misurato attraverso la canna e i palmi, non si conoscono gli anni a cui i prezzi riportati facciano riferimento. Sappiamo soltanto che 2 canne e 2 palmi di drappo costavano 3 1l. e 26 s. la canna.

Estopes: citate 33 volte. Si trattava della parte più grossa e spessa della canapa o del lino. Era un tessuto usato soprattutto nelle barche. Si utilizzava, 
inoltre, per tovaglie, lenzuola, camicie, mutande, coperte e asciugamani. La Marsà ricorda che questo tipo di stoffa veniva usata per avvolgere altri panni più preziosi come il drap de Pugcerdà. Quando veniva venduto come tale aveva, ovviamente, un prezzo più basso rispetto a quando veniva venduto da solo (Marsà Vila, 1977: 64). Gli unici dati sicuri che è possibile trarre dal registro riguardano gli anni 1335, 1337, tali per cui a giugno del 1335 l'estopes avevano un prezzo di $2 \mathrm{~s}$. per canna, che, come già detto in precedenza, era una misura che corrispondeva a 8 palmi e 6 piedi, il suo valore in metri, a Barcellona, corrispondeva a circa 1,55. Nel 1337 il prezzo varia secondo un calcolo al centinaio di canne costando $91110 \mathrm{~s}$.

Fera: È il ferro ed è citato dieci volte. Veniva pesato e venduto a fascine. Dal lavoro della Marsà ricaviamo il prezzo di vendita a Barcellona nel 1337, 1 11. e 12 s. barcellonesi per fascina (Marsà Vila, 1977: 64). Per Cagliari non abbiamo il prezzo di vendita per una fascina ma per quantità di fascine, così, 24 fascine costavano $4811.14 \mathrm{~s} .1 \mathrm{dr}$. per circa 2 ll. a fascina, ma è difficile stabilire con esattezza il prezzo essendo le fascine di peso variabile.

Flasades: citate ventidue volte nelle tipologie flasades di Girona, di Barcellona e d'Aragona. Secondo l'Alcover, si trattava di una pezza di tessuto quadrangolare di lana o cotone generalmente con righe di diversi colori, usata come coperta per i letti. Nella piazza di Castel de Càller, nel 1336, il prezzo di una flasada oscillava da 6 s. 2 dr. per pezza a 6 s. 3 dr., 6 s. 7 dr., 6 s. 6 dr. del 1337.

L'analisi dei commerci tra le due città, Barcellona e Cagliari, unitamente ad uno studio di tipo socio-amministrativo sono un primo risultato scaturito dallo studio del llibre de deu i deg. In questa sede si è voluto fornire solo un breve spaccato di tale libro.

Le conclusioni che fin qui si possono formulare sono parziali in quanto relative ad una sola fonte documentale, il llibre de deu i deg di Johan Benet, ad esse andranno aggiunte quelle sulle altre due fonti inedite della famiglia Benet, il Manual de comptes di Johan Benet, e il Llibre de comptes di Joan Bonet, di cui mi sto attualmente occupando nella mia tesi dottorale per l'Università di Saragozza, Doctorado en Historia, Sociedad y Cultura, épocas Medieval y Moderna sotto la guida del professor German Navarro Espinach.

L'obiettivo della futura tesi dottorale è, infatti, quello di mettere insieme, come in una collatio, le informazioni provenienti dai diversi libri di conto della 
famiglia Benet per ricavare auspicabilmente nuovi dati a beneficio sia della storia della Sardegna trecentesca, sia della storia mediterranea della Corona d'Aragona.

La scarsità delle fonti sarde, dovuta a distruzioni e dispersioni succedutesi nel tempo, ci pone sicuramente di fronte a problemi e incognite di vario genere, ma l'accrescimento del nostro patrimonio di conoscenze per l'epoca sarà già un passo rilevante.

Il percorso professionale di Johan Benet, si è dimostrato un esempio capace di illustrare le realtà sociali della città, dove il registro è stato scritto raccontando spaccati di vita personale tra le scarne registrazioni contabili. Nelle sue carte sono rimaste impresse la vita amministrativa e quella economica fatta di prestiti, di cambi, specchio del trend del grande commercio mediterraneo, di tasse in entrata o in uscita, dai cereali al vino, all'olio, di contratti di comanda, di compravendite, di importazioni ed esportazioni verso centri portuali che non sono soltanto catalani ma appartenenti a diverse città del centro e del meridione d'Italia. L'analisi degli altri libri di conti della famiglia Benet, avendo a disposizione datazioni diverse, permetterà di integrare l'enorme ricchezza di notizie ignorate, o appena accennate, collocandole nel giusto modo nell'insieme delle conoscenze storiche del medioevo mediterraneo e sardo in particolare. Se la peste del 1348 non fosse sopraggiunta a decimare quasi tutti i membri della famiglia, essa certamente sarebbe arrivata molto in alto nella scala sociale della città catalana e avremmo potuto avere a disposizione, forse, un numero molto maggiore d'informazioni!

Il registro de deu i deg di Johan Benet ci presenta una contabilità di partita semplice, registrata mettendo in evidenza soprattutto le informazioni utili a tenere memoria dei debiti o dei crediti e con una tale quantità di conti che da soli ci danno la dimensione della complessità e della portata degli affari dei Benet. Quest'ultimo libro di Johan Benet termina le registrazioni nel giugno del 1338 mentre il Manual de comptes le inizia a luglio del medesimo anno rappresentando un interessante esempio di continuità negli affari del mercante. Lo studio, in corso, dei successivi registri contabili ha già messo in evidenza la stretta relazione che la famiglia intratteneva con la città di Castel de Cáller. Un dato ulteriore, emerge in maniera sempre più netta; i libri forniscono, infatti, delle registrazioni che segnano, o meglio, disegnano l'itinerario seguito dalle merci, a partire dal luogo in cui venivano comprate fino al luogo di vendita con un corredo piuttosto interessante di costi che venivano sostenuti per l'acquisto, 
il trasporto, l'imballaggio, i diritti doganali e i prezzi, tutti registrati con meticolosità e precisione. ${ }^{45}$

Anche le merci finora incontrate si soffermano prevalentemente sui tessuti importati da Barcellona che catalizzava su di sé una grande concentrazione di prodotti provenienti dall'entroterra catalano, dal sud della Francia, dalle Fiandre e per restare in Italia i commerci, come già emerso nel precedente libro studiato, si concentrano su Cagliari, Napoli e la Sicilia.

Il Manual de comptes e il Llibre de comptes confermano ciò che già è stato detto per il Llibre de deu i deg, di cui si è appena parlato, e ne rendono più ricche le informazioni con l'aggiunta di nuove figure di mercanti e artigiani che abitavano nel Castel e, non secondario, l'allungamento dell'arco cronologico, dal 1332 al 1356, considerando i registri nella loro totalità.

Lo studio completo dei libri renderà possibile integrare le nostre conoscenze con l'apporto di nuovi dati bibliografici relativi alle altre piazze commerciali della Corona d'Aragona permettendo uno studio comparato che apporterà dati sicuramente interessanti utili a mettere a fuoco in maniera più precisa il quadro economico del medioevo mediterraneo della prima metà del secolo XIV.

\section{Riferimenti bibliografici}

ALCOVER, A.M. - MOLL, F. de B. (1930-62), Diccionari català-valencià-balear, 10 voll., Palma de Mallorca.

ALIAS F. (2008-2009), Rendita e fiscalità nel Regno di Sardegna (prima metà del Trecento). Tesi di Dottorato in "Antropologia, Storia Medioevale, Filologia e Letterature del Mediterraneo Occidentale in Relazione alla Sardegna". Ciclo XXI. Università degli Studi di Sassari, a.a. 2008-2009.

ALSINA, C. - FELIU, G. - MARQUET, L. (1990), Pesos, mides i mesures dels països catalans, Barcelona, Curial, 130-131, 188-189.

ARRIBAS PALAU A. (1952), La conquista de Cerdeña por Jaume II de Aragón, Barcelona, Instituto Español de Estudios Mediterráneos, 55, 189 e segg.

ARTIZZU F. (1961-62), Documenti inediti relativi ai rapporti economici tra la Sardegna e Pisa nel Medioevo, vol. II, Padova, CEDAM, 14-21.

ARTIZZU F. (1958), Rendite pisane nel Giudicato di Cagliari agli inizi del Secolo XIV, Padova, Cedam.

BOSCOLO A. (1973), Documenti sull'economia e la società in Sardegna all'epoca di Alfonso il Benigno, Padova, docc. n. 240, n. 239.

45 Ulteriori precisazioni e approfondimenti saranno oggetto della tesi dottorale, pertanto, per successivi sviluppi su questi argomenti si dovrà attendere la stesura finale della ricerca. 
CADINU M. (2008), I «l nuovo quartiere Aragonese sul porto nel primo Trecento a Cagliari», in M. Cadinu e E. Guidoni (a cura di), La città europea del Trecento: trasformazioni, monumenti, ampliamenti urbani, Atti del Convegno internazionale, Cagliari, 9-10 dicembre 2015, Roma, Kappa, 81-101.

CASULA F.C. (1990), La Sardegna Aragonese, Sassari, Chiarella, 61-70.

CONDE R. (1988), «Las actividades y operaciones de la banca barcelonesa trecentista de Pere Descaus y Andreu d'Olivella», Revista Española de Financiación y Contabilidad, 55, vol. XVII, Madrid, 128.

DAY J. (1978), «The decline of a money economy: Sardinia in the late Middle Age», in Studi in memoria di Federigo Melis, Napoli, 1978, vol. III, 156.

DE ROOVER R. (1948), Money, banking and credit in mediaeval Bruges: Italian merchant-bankers Lombards and money-changers- A study in the origins of banking, The Mediaeval Academy of America, Cambridge.

FIGUS S. (2012-2013), I commerci tra Barcellona e Cagliari nella prima metà del secolo XIV attraverso il Llibre de deu i deg di Johan Benet, 1334-1338, ACB, Extravagants, Comptes de particulars, companyes etc., tesi di laurea magistrale, relatore prof. Sergio Tognetti, Università degli Studi di Cagliari (2012-2013).

FIGUS S. (2015), «Il Castel de Caller catalano-aragonese quale appare dal Llibre de deu i deg di Johan Benet, mercante di Barcellona», RiME, n. 15/2, dicembre 2015, 271-311.

GARCÍA SANZ, A. (1964-67), El contrato de la dita y la letra de cambio, Ausa 5, Vic, 81-87.

MADURELL MARIMON, J.M.-GARCIÁ SANZ, A. (1973), Comandes comercials barceloneses de la Baixa Edat Mitjana, Barcelona, 107-114; 204-205.

MANCA C. (1969), Il libro di conti di Miquel Ça Rovira, Padova, CEDAM.

MANCA C. (1966), Aspetti dell'espansione economica catalano-aragonese nel Mediterraneo occidentale. Il commercio internazionale del sale, Milano, Giuffrè, 114-117.

MANCA C. (1967), Fonti e orientamenti per la storia economica della Sardegna Aragonese, Padova, Cedam.

MARSÁ VILA M. (1977), Algunos elementos para el estudio de las relaciones comerciales entre Cagliari y Barcelona en la primera mitad del siglo XIV ( «Libres de compres $i$ vendes de Johan Benet, Càller, 1332-1338»). Tesis de Licenciatura, Universitad de Barcelona.

MELIS F. (1972), Documenti per la storia economica dei secoli XIII-XVI, con una nota di paleografia commerciale a cura di Elena Cecchi, Olschki, Firenze, 1972, 84-85; 104-119.

OLIVA A. M. (1980), «Algunos elementos para el estudio de la relaciones entre Cagliari y Barcelona en la primera mitad del siglo XIV (Libre de compres i vendes de Johan Benet. Caller, 1332-1338)», Medioevo. Saggi e Rassegne, n.5, 65-103. 
OLLA-REPETTO G. (1969), Gli ufficiali regi di Sardegna durante il regno di Alfonso IV, Fossataro, Cagliari, 1969, 3-47.

PETRUCCI S. (2005-2006), Cagliari nel Trecento. Politica, istituzioni, economia e società. Dalla conquista aragonese alla guerra tra Arborea ed Aragona (13231365). Tesi di Dottorato in 'Antropologia, Storia medievale, Filologia e Letterature del Mediterraneo Occidentale in relazione alla Sardegna' (XX ciclo), Università degli Studi di Sassari a.a. 2005-2006.

PINNA M. (1928), «Le Ordinazioni dei Consiglieri del Castello di Cagliari del secolo XIV», Archivio Storico Sardo, vol. XVII, ordinazione n. 30.

RIU M. (1964), «Aportación a la organización gremial de la industria textil catalana en el siglo XIV», VII Congreso Historia de la Corona de Aragón, II, Barcelona, 548-549.

SALAVERT Y ROCA V. (1956), Cerdeña y la expansión mediterranea de la Corona de Aragón, 1297-1314, Consejo Superior de Investigaciones Científicas, Escuela de Estudios Medievales, Madrid, 114-125.

SIMBULA P. F. (2000), Gli Statuti del Porto di Cagliari (secoli XIV-XVI), AM\&D, Cagliari, 2000.

TANGHERONI M. (1989), «Aspetti economici dell'espansione catalano-aragonese nel Mediterraneo», in G. Olla-Repetto (a cura di), La Corona d'Aragona: un patrimonio comune per Italia e Spagna (secc. XIV-XV), Cagliari, Cittadella dei Musei, 27 gennaio-1 maggio 1989, Deputazione di Storia Patria per la Sardegna, $50-65$.

TANGHERONI M. (1981), Aspetti del commercio dei cereali nei paesi della Corona d'Aragona. I. La Sardegna, Pisa, Pacini.

TANGHERONI M. (1996), Commercio e navigazione nel Medioevo, Laterza, RomaBari, 341-350.

TANGHERONI M. (1983), «Le coste nel Mediterraneo Medievale: alterne vicende della vita e dell'economia», in A. Asole (dir.), Sardegna: l'uomo e le coste, Sassari, Banco di Sardegna, 43-54.

TANGHERONI M. (1973), Politica, commercio, agricoltura a Pisa nel Trecento, Pacini, Pisa (riedizione aggiornata: 2002), 119-126.

\section{Fonti documentali}

ACB, Llibres extravagants, Comptes de particulars, Llibre de deu y deig di Joan Benet 1334-1338.

ACB, Llibres extravagants, Comptes de particulars, Manual de comptes di Johan Benet, 1338-1344.

ACB, Llibres extravagants, Comptes de particulars, Llibre de comptes di Joan Bonet, 1332-1356.

ACB, Llibres extravagants, Comptes de particulars, Libre de compres $i$ vendes di Johan Benet, Càller, 1332-1338. 
ACA, Cancilleria, Reg. 514, f. 286r-v (1332, dicembre 28).

ACA, Cancilleria, Reg. 512, f. 280r (1331, ottobre 25).

ACA, Real Patrimonio, Reg. 2060, f. 71r; f. 97r; f. 75v; f. 82v; f. 93v.

ASCA, AAR, B5

Risorse Elettroniche

$<$ http://dcvb.iecat.net/>

$<\mathrm{http}: / /$ www.enciclopedia.cat/> 